\title{
Anabases
}

ANABASES Traditions et réceptions de l'Antiquité

$8 \mid 2008$

Varia

Richard HUNTER, The Shadow of Callimachus. Studies in the reception of Hellenistic poetry at Rome, Roman literature and its contexts

\section{Maxime Pierre}

\section{OpenEdition}

Journals

Édition électronique

URL : http://journals.openedition.org/anabases/256

DOI : 10.4000/anabases.256

ISSN : 2256-9421

Éditeur

E.R.A.S.M.E.

Édition imprimée

Date de publication : 1 octobre 2008

Pagination : 293-294

ISSN : 1774-4296

\section{Référence électronique}

Maxime Pierre, "Richard Hunter, The Shadow of Callimachus. Studies in the reception of Hellenistic poetry at Rome, Roman literature and its contexts », Anabases [En ligne], 8 | 2008, mis en ligne le 01 juillet 2011, consulté le 22 septembre 2020. URL : http://journals.openedition.org/anabases/256 ; DOI : https:// doi.org/10.4000/anabases. 256

Ce document a été généré automatiquement le 22 septembre 2020

(c) Anabases 


\title{
Richard HUNTER, The Shadow of Callimachus. Studies in the reception of Hellenistic poetry at Rome, Roman literature and its contexts
}

\author{
Maxime Pierre
}

\section{RÉFÉRENCE}

Richard HUNTER, The Shadow of Callimachus. Studies in the reception of Hellenistic poetry at Rome, Roman literature and its contexts, Cambridge (GB), Cambridge University Press, 2006, $162 \mathrm{p}$.

21,56 euros / ISBN 978-0-521-87118-1.

1 Ce volume de Richard Hunter qui comprend quatre études sur la réception de Callimaque à Rome participe du renouveau des études de la poésie latine dans le domaine anglo-saxon inauguré dans cette même collection par le travail de Stephen Hinds sur l'intertextualité (Stephen Hinds, Allusion and intertext: dynamics of appropriation in Roman poetry, Cambridge, Cambridge University Press, 1998). L'ouvrage se donne ici pour but de contribuer au débat contemporain sur l'hellénisation en analysant les points de continuité et de rupture avec la poésie alexandrine. Tout en proposant un objet d'étude somme toute classique depuis l'ouvrage de Walter Wimmel (Kallimachos in Rom, Wiesbaden, Steiner, 1960) qui a connu un retentissement important dans le champ des études littéraires, Richard Hunter, invite à explorer l'influence de textes et de thèmes moins connus de l'alexandrinisme.

2 L'auteur commence son étude par l'exploration d'un topos alexandrin d'origine hésiodique : le "bois sacré». Il s'agit du bois sacré de l'Hélicon qui est utilisé par Callimaque comme lieu de rencontre et de transmission poétique. Il sera repris comme tel par Properce, Ovide et Horace : plus spécifiquement à Rome, le topos du bois sacré 
est représenté comme le lieu de la transmission poétique, où le poète romain vient prendre la suite des poètes grecs. Il s'agirait, suivant la thèse développée, d'un lieu décisif, où le poète s'engage symboliquement sur une voie poétique : le choix de l'élégie pour Properce et Ovide. Richard Hunter, poursuit son analyse en abordant la figure de Dionysos, envisagé, au travers d'une relecture de l'Ion de Platon comme divinité de l'inspiration. Ce faisant, l'auteur relève une source plus spécifiquement alexandrine de l'utilisation de Dionysos par les Romains : l'usage - notamment chez Callimaque dans l'Hymne à Délos (165-170) - d'un Dionysos-Osiris conquérant qui sert de référence pour représenter les conquêtes d'Alexandre puis des Ptolémées. Ce thème ptolémaïque de conquête du monde se trouve en effet chez Tibulle pour fêter le triomphe de Messala (I, 7) mais également chez Horace, Properce et Virgile pour célébrer l'extension de l'empire par Auguste. Le troisième chapitre de l'étude passe d'une analyse thématique à un point de vue stylistique, en analysant l'usage poétique de la comparaison. En effet, cette figure, utilisée depuis Homère, est retravaillée à Alexandrie pour produire des effets inédits : la comparaison devient le lieu par excellence de tours de force poétiques et de jeu avec la tradition, consistant par exemple à inverser le comparant et le comparé ou à faire allusion à un intertexte. L'usage alexandrin de cette figure est repris à Rome comme véritable marqueur d'intertextualité. La comparaison permet ainsi des jeux de réflexivité: si du point de vue littéral elle crée une analogie entre deux référents fictifs, du point de vue de la réflexivité, elle invite à comparer le texte aux précédents. La réflexion sur l'intertextualité et la réflexivité de la poésie augustéenne est prolongée dans le dernier chapitre où les Bucoliques de Virgile sont mises en perspective avec la tradition alexandrine. Hunter considère ici la manière dont Virgile thématise l'appropriation romaine de la poésie grecque : l'image des champs conquis par le militaire (I, 70-71) ou la colombe saisie par les aigles (IX, 13) seraient des images réflexives renvoyant à l'appropriation du genre bucolique par les Romains, anticipant le fameux Graecia capta ferum uictorem cepit d'Horace (Ep. I, 2, 156). En outre, les destinataires des Bucoliques seraient ici assimilés aux princes lagides : il s'agirait pour Virgile de renouveler à Rome l'idéologie pacifique de Ptolémée Philadelphe célébrée par Théocrite.

3 Malgré leur hétérogénéité, les quatre chapitres accomplissent leur objectif : montrer sous un autre angle la réception de Callimaque à Rome. Nous retiendrons en particulier cette idée stimulante que l'adoption du modèle alexandrin n'obéit pas tant à un programme littéraire qu'à un programme politique. Ainsi l'étude démontre-t-elle que le callimaquisme n'est pas simplement une esthétique, comme on s'est longtemps plu à le décrire, mais fait partie d'un vaste système idéologique qui passe à Alexandrie par la réappropriation d'un patrimoine culturel au profit de la dynastie des Ptolémées. Le système augustéen répète le geste des Lagides : il s'agit à nouveau de la réappropriation par Rome de la culture grecque. Une telle analyse n'est pas inédite; d'autres latinistes, en particulier en France, Alain Deremetz (Le Miroir des Muses, poétiques de la réflexivité à Rome, Presses Universitaires du Septentrion, 1995) ont déjà montré la manière dont l'intertextualité et la réflexivité étaient, dans la poésie de Properce, Virgile, et Horace, mises au service d'une "refondation» augustéenne. Le mérite de ce livre est d'approfondir cette thèse avec de nouveaux textes et de nouvelles perspectives. 


\section{AUTEUR}

\section{MAXIME PIERRE}

Paris VII-Gênes

Maxime.pierre@unige.it 